\title{
Pharmacokinetics of Lithium in Egyptian Bipolar Patients: Dosage Adjustment Approach
}

\author{
Abdel-Hameed Ibrahim Mohamed Ebid', Dina Awny Tawfik Abd-Allah', \\ Mahmoud Mamdouh Mohamed Elhabiby² \\ ${ }^{1}$ Pharmacy Practice Department, Faculty of Pharmacy, Helwan University, Cairo, Egypt \\ ${ }^{2}$ Psychiatry Department, Faculty of Medicine, Ain-Shams University, Cairo, Egypt \\ Email: dina awny 88@hotmail.com
}

Received 24 February 2014; revised 25 March 2014; accepted 11 April 2014

Copyright (C) 2014 by authors and Scientific Research Publishing Inc.

This work is licensed under the Creative Commons Attribution International License (CC BY). http://creativecommons.org/licenses/by/4.0/

c) (i) Open Access

\begin{abstract}
Due to its proven clinical effectiveness, lithium has been considered as a corner stone for the long-term treatment of Bipolar Disorder (BPD) for more than half a century. The objective of this study was to evaluate the effect of patients' different co-variables on lithium pharmacokinetics and the development of a pharmacokinetic model for the estimation of lithium clearance in Egyptian bipolar patients; this model can be used afterwards during dosage adjustment to achieve target's steady-state plasma concentrations in similar settings. The study was conducted on 65 adult Egyptian bipolar patients of both genders, in both the in- and out-patient settings in the Institute of Psychiatry, Faculty of Medicine, Ain Shams University, Cairo, Egypt. Patients' full profiles were prepared, containing all their data. Single daily dose regimen was followed by all patients and one blood sample was drawn $12 \pm 0.5$ hours post dose for steady-state lithium concentration, sodium level and serum creatinine determinations. Lithium trough levels were determined using Flame Emission Photometry technique. Data analysis and regression modeling revealed a significant correlation between total body weight (TBW), body surface area (BSA) and lithium clearance. Due to multicollinearity, BSA was excluded from the basic non-linear regression equation and TBW was found to be the only covariate affecting lithium clearance. Mean lithium clearance was found to be $0.243 \pm 0.097 \mathrm{Lit} / \mathrm{Kg} / \mathrm{day}$, ranging from 0.091 to $0.71 \mathrm{Lit} / \mathrm{Kg} / \mathrm{day}$.
\end{abstract}

\section{Keywords}

Lithium; Pharmacokinetics; Bipolar Disorder 


\section{Introduction}

Bipolar disorder is considered to be a serious psychiatric illness that should be paid a great attention for its great effect on the economy and society [1]. It's characterized by both poles of mania and depression episodes in several forms and degrees of severity [2]. It has been proven that BPD is a far more serious mental illness than previously thought due to high suicidal rate, great functional impairments, and its chronic nature [3]. Mood disturbances are most prominent in the acute manic and depressive episodes, but they still exist during remission periods [4] [5]. Precipitating factors of BPD include psychosocial stress that affects brain functions deeply and contributes to depressive and anxious symptoms [6] [7], changes in sleep-wake schedule or sleep deprivation, which may exacerbate manic episodes [8] and also ischemia, hypoglycemia and excitatory amino acid toxicity, as they decrease the expression of neurotrophic factors necessary for the neuronal viability [9].

Since its discovery by Cade [10], lithium has proven a remarkable efficacy as a mood stabilizer [11], antisuicidal, neuroprotective and anti-oxidative [12]. The pharmacokinetic properties of lithium have been previously studied on both healthy and bipolar patients, mostly using lithium carbonate salt [13]. The time for maximum concentration $t_{\max }$ was found to range from 1.0 to 2.0 hours and from 4.0 to 5.0 hours for standard release and sustained release preparations, respectively. Bioavailability (F) ranges from $80 \%-100 \%$ while volume of distribution $\left(V_{d}\right)$ value was estimated to be $0.7-1.0 \mathrm{~L} / \mathrm{kg}$. A total body clearance of lithium was $10-40 \mathrm{~mL} / \mathrm{min}$ with high inter-individual variability; it increases as much as $50 \%$ during an acute manic episode producing a half-life that is $1 / 2$ of the normal value. Lithium is exclusively excreated through the kidney as a free ion without any metabolic transformation [14]-[16]. Normally, terminal elimination half-life $\left(t \frac{1}{2} \beta\right)$ is 18 - 36 hours, while absorption half-life is $3.73 \pm 0.37$ hours for sustained release forms of lithium carbonate [17]. Concomitant ingestion of food tends to increase lithium absorption [18].

The narrow therapeutic range of lithium $(0.6-1.2 \mathrm{mmol} / \mathrm{Lit})$ and the wide inter-individual variability in its clearance have led to the development of several dosage regimens [19] [20], that's why we are taking the patients' co-variables (Age, Gender, TBW, Height, BSA, Sodium Level and Kidney Functions) into consideration for better effectiveness and lower toxicity incidence. Normally lithium toxicity occurs at serum lithium concentrations greater than 1.5 - $2.0 \mathrm{mmol} / \mathrm{Lit}$, although sometimes it can be present at "therapeutic levels" (usually in the elderly patients due to their reduced clearance) [21]. The features of lithium toxicity include impaired consciousness (ranging from drowsiness to coma), apathy, hyper-reflexia, hypertonia, muscle fasciculations, dysarthria, ataxia, coarse tremor, myoclonus, choreoathetoid movements, seizures and spasticity, cardiac dysrhythmias, acute renal failure [22], tinnitus, blurred vision and nystagmus, respiratory complications and irreversible brain damage [15]. Levels greater than $3.5 \mathrm{mmol} / \mathrm{Lit}$ are potentially lethal and hemodialysis is recommended [23].

\section{Patients and Methods}

\subsection{Patients' Selection}

The study was conducted on patients suffering from Bipolar Disorder (According to DSM-IV Criteria [8]); selected from the in- and out-patient settings of the Institute of Psychiatry, Faculty of Medicine-Ain Shams University; Cairo, Egypt during the period of June 2013 to December 2013 after approval of the Hospital Medical Ethical Committee. A patient is initially considered to be a candidate for this study when lithium therapy was indicated. Medical history, careful physical examination, demographics and complete investigations were obtained upon enrolment into the study, with special focus on kidney functions.

Patients involved in our study were adult Egyptian patients suffering from Bipolar Disorder and were regularly on lithium therapy; Prianil C. ${ }^{\circledR} 400 \mathrm{mg}$ tablets, with available complete data about their lithium dosage and administration time. Two weeks at least must have been passed from last dosage adjustment; to make sure that lithium levels had reached a steady state, with no known medical problem causing marked dysfunction for patients' health.

The patients were excluded primarily if the time of the dose administration was inaccurate or unknown, poor compliance assessed by the regular interviewer of the patient or in case of active hallucinations or delusions. All study participants provided written and signed informed consent before any related procedures were performed; patients were excluded when they had refused this consent. Baseline interviews were conducted for selection, 
evaluation and improvement of compliance. Compliance was assessed by asking the patient or his/her relatives about regular use of medications and how long the supply of medicines had lasted. The selected patients comprised 43 (66.2\%) males and 22 (33.8\%) females with a mean age of $34.785 \pm 11.162$ years.

\subsection{Treatment Regimen and Data Collection}

Each patient was kept on a stable lithium carbonate, Prianil C.R. ${ }^{\circledR} 400 \mathrm{mg}$ tablets, dosage regimen either 200, $400,600,800$ or $1000 \mathrm{mg}$ once daily for more than one month so as to ensure that the measured concentration was at steady state. The duration of treatment was determined by the attending physician during the routine interview on the basis of clinical status and laboratory results. On the day of sampling, patients' demographic data were collected regarding gender, age in years, TBW in $\mathrm{kg}$, height in $\mathrm{cm}$, lithium dosing regimen in $\mathrm{mg} /$ day, serum lithium concentration in steady state (mmol/Lit), serum sodium level $(\mathrm{mEq} / \mathrm{Lit})$ and serum creatinine in $\mathrm{mg} \%$. Additional clinical factor (BSA in $\mathrm{m}^{2}$ ) was calculated according to the Mosteller formula [24] as shown in Equation (1):

$$
\text { BSA }=\sqrt{\frac{\text { Weight }(\mathrm{Kg}) \times \text { Height }(\mathrm{cm})}{3600}}
$$

\subsection{Blood Sampling and Assay}

The blood samples were drawn from a peripheral vein from each patient; $12 \pm 0.5$ hours post dose ( $C_{S S}$ trough) [15], with a sterilized plastic disposable syringe and were used for steady state lithium serum determination and other laboratory tests. Blood samples were clotted at room temperature and centrifuged at 3000 revolution per minute (rpm) for 10 minutes. The separated serum was stored frozen at $-20^{\circ} \mathrm{C}$ till analysis according to Tietz [25]. Lithium serum drug levels were measured by Flame Emission Photometry technique using the standard; supplied by HOSPITEX DIAGNOSTICS s.r.l. Via Provinciale Lucchese, Sesto Fiorentino, FIRENZE-ITALY, marketed as (Standard Na/K/Li 140/5/1 mEq/L).

The Flame Emission Photometry instrument was a Sherwood-Corning 410, which was equipped with an automatic diluter. The instrument was calibrated with Multical standard $(1.5 \mathrm{mmol} / \mathrm{L} \mathrm{Li}$ concentration) solution and neat pool control serum samples were introduced to the instrument.

\subsection{Pharmacokinetic Analysis}

Lithium follows two-compartment open model, with a first order elimination. A very simple pharmacokinetic equation that computes the average lithium steady-state serum concentration $\left(C_{S S}\right.$ in $\left.\mathrm{mmol} / \mathrm{L}\right)$ is widely used and allows maintenance dosage calculation [26] as shown in Equation (2):

$$
C_{S S}=[F(D / \tau)] / \mathrm{Cl}
$$

where, $F$ is the bioavailability, fraction for the oral dosage form ( $F=1$ for oral lithium), $D$ is the lithium dose in milli-moles, $\tau$ is the dosage interval in days, $C_{S S}$ is the steady-state lithium concentration, and $\mathrm{Cl}$ is lithium clearance in liters per day (L/day). Because this equation computes lithium ion requirement and lithium carbonate doses are prescribed in milligrams, the ratio of lithium ion content to lithium carbonate salt $(8.12 \mathrm{mmol}$ $\mathrm{Li}^{+} / 300 \mathrm{mg}$ lithium carbonate) is used to convert the result from this equation into a lithium carbonate dose [15].

Using the $\boldsymbol{X L S T A T E}{ }^{\circledR}$ pro 2013.6.02 program, the individual effect of gender on clearance was studied using Mann Whitney test for comparison between two independent means. $P$-value was significant at $\leq 0.05$ level. Also the individual effect of age, TBW, height, sodium level, serum creatinine, lithium daily dose and steady state drug level on clearance was studied using correlation analysis (Spearman Rho test). $P$-value was significant at $\leq 0.05$ level.

Multiple regression analysis using GraphPad InState ${ }^{\circledast}$ software package, V. 3.05, 2000 program was used to drive models describing the dependence of clearance on the studied covariates. The equation of the basic (first) model of clearance was deduced by the program after feeding all of the studied covariates of each patient. The program evaluated the effect of removal of each covariate from the basic model of clearance to reach the next model of clearance by determining which variables made a significant contribution in the model as the $P$-value, for each variable, compared a full model of variables with a simpler model omitting one variable. It tested the 
effect of one variable after accounting for the effects of the others. Only covariates which led to a significant contribution were retained to develop the next model of clearance.

Multicollinearity of co-variables was evaluated as the R squared (variance), for each variable, quantified how well each variable was predicted from the other variables to ensure keeping all variables independent of each other so as to exclude dependent variables.

The equation of the next model of clearance was deduced by the program after feeding covariates of each patient which led to a significant contribution only and the program repeated the process of evaluating the effect of removal of each covariate from the model to test its significant contribution until reaching to the final model of clearance.

Clearance values for each observation can be predicted and the residual values can be calculated in all models of clearance. To assess the validation of prediction of clearance values for each observation, a paired-comparisons t-test was run between observed and predicted clearance values.

\section{Results}

\subsection{Patients}

The studied group comprised 65 adult bipolar Egyptian patients, whose demographic and clinical data are shown in Table 1. The Lithium carbonate, Prianil C.R. ${ }^{\circledR} 400 \mathrm{mg}$ tablets, was received orally by different dosage regimens starting from $200 \mathrm{mg}$ to $1000 \mathrm{mg}$ once daily at night, with a mean of $752 \pm 125 \mathrm{mg} / \mathrm{day}$. To convert the obtained data about lithium daily dosage from milli-grams lithium carbonate into a mmol $\mathrm{Li}^{+}$daily dose, Equation (3) is used [15].

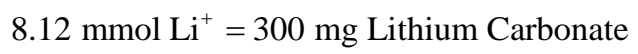

Data of lithium therapy and laboratory findings are detailed in Table 2.

\subsection{Model Building Procedures}

In the beginning, we performed an initial screening to give a first impression of the relationships between covariates and lithium clearance. To carry out this step individual estimates of clearance were first obtained and subsequently the significance of each possible covariate as an affecting parameter was evaluated. The covariates evaluated were gender (using Mann-Whitney $U$ test) and age, TBW, height, BSA, sodium level and serum creatinine ( $\mathbf{S r} \mathbf{C r}$ ) (using Spearman's Rho correlation test). Summary of the effect of studied patients' co-variables on lithium clearance is explained by Table 3. Gender wasn't found to be significantly affecting lithium clearance; the $P$ value resulted was 0.667 which is considered non-significant ( $>0.05)$.

After that, the basic model was created by incorporating all covariates and testing them. The basic model of lithium clearance $(\mathrm{Cl})$ was found to be as shown in Equation (4)

$$
\begin{aligned}
\mathrm{Cl}= & 0.8650+0.001070 \times \text { Age }-0.003017 \times \mathrm{TBW}+0.0005230 \times \mathrm{Ht} \\
& +0.01328 \times \mathrm{BSA}-0.003637 \times \mathrm{Na} \text { Level }-0.03689 \times \mathrm{Sr} \mathrm{Cr}
\end{aligned}
$$

\begin{tabular}{|c|c|c|c|}
\hline Characteristics & Mean \pm SD & Range & CV\% \\
\hline Number of patients & 65 & & \\
\hline Gender (male/female), \% & (43/22), (66.2\%/33.8\%) & & \\
\hline Age (years) & $34.785 \pm 11.162$ & $18-65$ & $32.1 \%$ \\
\hline TBW (Kg) & $80.211 \pm 16.925$ & $42-121.5$ & $21.1 \%$ \\
\hline Height (cm) & $166.292 \pm 10.225$ & $140-190$ & $6.148 \%$ \\
\hline $\operatorname{BSA}\left(\mathbf{m}^{2}\right)$ & $1.915 \pm 0.221$ & $1.278-2.451$ & $11.564 \%$ \\
\hline Serum creatinine (mg\%) & $0.813 \pm 0.134$ & $0.49-1.10$ & $16.4978 \%$ \\
\hline Sodium level (mEq/Lit) & $137.415 \pm 3.754$ & $126-149$ & $2.7315 \%$ \\
\hline
\end{tabular}

Table 1. Demographic and clinical characteristics of enrolled patients $(n=65)$. 
Table 2. Lithium steady state concentrations, dosage regimens and clearance of patients $(n=65)$.

\begin{tabular}{cccc}
\hline Parameter & Mean \pm SD & Range & CV\% \\
\hline Lithium dose (mg/day) & $735.385 \pm 145.106$ & $200-1000$ & $19.732 \%$ \\
Lithium dose (mmol/Kg/day) & $0.260 \pm 0.074$ & $0.052-0.433$ & $28.622 \%$ \\
Lithium trough Css (mmol/Lit) & $1.145 \pm 0.335$ & $0.3-2.4$ & $29.279 \%$ \\
Li clearance (Lit/Kg/day) & $0.243 \pm 0.097$ & $0.091-0.71$ & $40 \%$ \\
\hline
\end{tabular}

Table 3. Summary of the correlation between patients' studied parameters and lithium clearance.

\begin{tabular}{|c|c|c|c|}
\hline Parameter & Spearman $\mathrm{r}^{\alpha}$ & $P$-value & Significance \\
\hline Age(Years) & 0.0770106 & 0.541 & $>0.05$, Non-Significant \\
\hline TBW (Kg) & -0.600881 & $<0.0001$ & $<0.01$, Significant \\
\hline Height(cm) & -0.087029 & 0.48967 & $>0.05$, Non-Significant \\
\hline BSA $\left(\mathbf{m}^{2}\right)$ & -0.57551 & $<0.0001$ & $<0.05$, Significant \\
\hline Serum creatinine (mg\%) & -0.22713 & 0.06897 & $>0.05$, Non-Significant \\
\hline Sodium level (mEq/Lit) & -0.110714 & 0.37904 & $>0.05$, Non-Significant \\
\hline
\end{tabular}

The mean of the differences between observed and predicted lithium clearance values did not differ significantly from zero. The two-tailed $P$ value was $0.9951(>0.05$, considered non-significant). R squared equaled $31.75 \%$; which is the percent of variance in $\mathrm{Cl}$ explained by the model. The $P$-value was 0.0008 , which is considered to be extremely significant. But multicollinearity was found between TBW and BSA and this meant that those variables provide redundant information, making the confidence intervals very wide. So we considered removing BSA as a variable from the basic model to avoid the multicollinearity and a reduced form of the basic model was deduced as follows on Equation (5)

$$
\begin{aligned}
\mathrm{Cl}= & 0.8644+0.001073 \times \text { Age }-0.002860 \times \mathrm{TBW}+0.0006013 \times \mathrm{Ht} \\
& -0.03652 \times \mathrm{Sr} \mathrm{Cr}-0.003636 \times \text { Na Level }
\end{aligned}
$$

The mean of the differences between observed and predicted values did not differ significantly from zero. The two-tailed $P$-value was $0.9963(>0.05$, considered non-significant). R squared equaled $31.75 \%$ and the $P$-value was 0.0003; considered extremely significant. Multicollinearity is not a problem anymore and the GraphPad InState $^{\circledR}$ program evaluated the effect of removal of each covariate from the reduced basic model of clearance to reach the next model of clearance by determining which variables made a significant contribution in the model as the $P$-value, for each variable, compared a full model of variables with a simpler model omitting one variable.

Only covariates which led to a significant contribution were retained to develop the next model of clearance. Our results revealed that TBW was the covariate of the significant contribution as shown in Equation (6)

$$
\mathrm{Cl}(\mathrm{Lit} / \mathrm{Kg} / \text { Day })=0.4871-0.003044 \times \mathrm{TBW}(\mathrm{Kg})
$$

The mean of the differences between observed and predicted values did not differ significantly from zero. The two-tailed $P$ value was 0.9964 ( $>0.05$, considered non-significant). R squared $=28.07 \%$ and the $P$-value was $<0.0001$; considered extremely significant. Correlation between lithium clearance and TBW is pointed out in Figure 1.

\section{Discussion}

Lithium Pharmacokinetics are influenced by a number of factors such as renal function [27], TBW [28], circumstances that affect fluid and electrolyte handling by kidney (i.e. hydration status, serum sodium level, concomitant medications and pregnancy) [29] [30] and age [16]. That's why in our study we were interested in finding the variables affecting clearance of lithium in bipolar Egyptian patients in order to improve their dosing regimens. So our approach to investigate the influence of demographic parameters and clinical data on lithium 


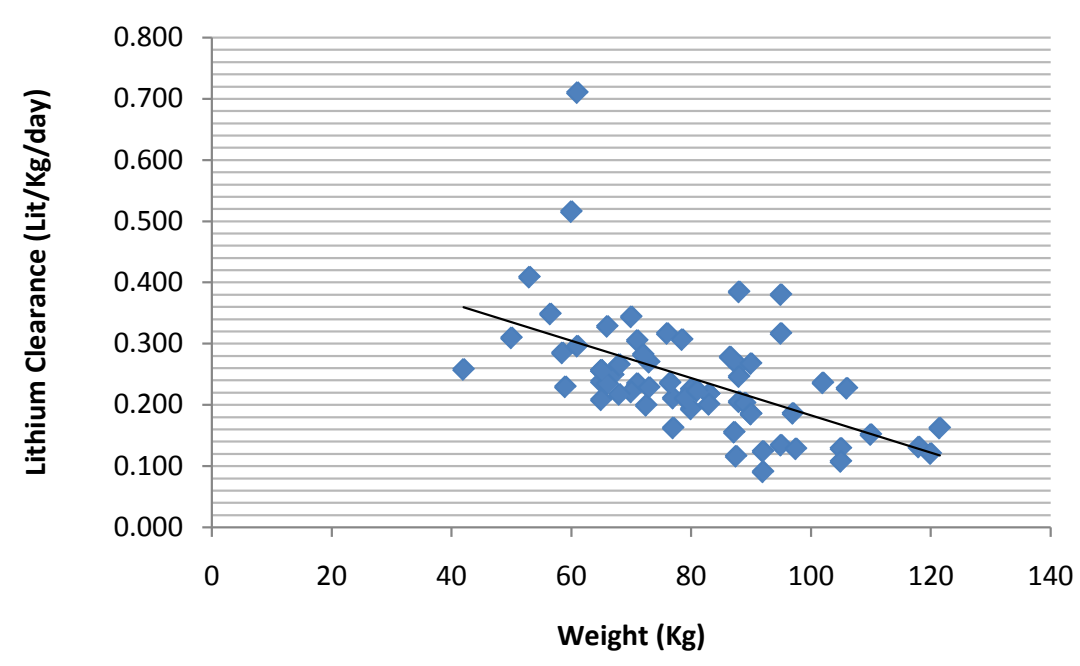

Figure 1. The effect of total body weight TBW on lithium clearance (Lit/kg/day).

clearance in Egyptian population yielded a regression model (Equations (4)-(6)) which relates clearance to these covariates. The estimated clearance of lithium was found to be (Mean \pm SD) $0.243 \pm 0.097 \mathrm{Lit} / \mathrm{kg} / \mathrm{day}$ with a wide range from 0.091 to $0.71 \mathrm{Lit} / \mathrm{kg} / \mathrm{day}$. These results are comparable to literature data, however; the differences in age, weight, dosage and analysis method of each study make it complicated to compare the absolute clearance indifferent ethnic groups.

The fitness of the basic model of clearance was the highest fit in all models ( $\mathrm{R}$ squared $=31.75 \%$ ) and best precision of modeling (RMSE $=0.084982$ ); this is an evidence of the importance of patient specific clinical data and demographics for better prediction of lithium clearance. Then GraphPad InState ${ }^{\circledR}$ program evaluated multicollinearity of variables in the model and the correlation matrix indicated correlation $(>0.9)$ between total body weight and body surface area; multicollinearity was a problem, so a reduced form of the basic model was deduced by removing $\boldsymbol{B S A}$ from modeling. The reduced basic model of clearance was also with the highest fit (R squared $=31.75 \%$ ) but without a multicollinearity problem.

Then the program deduced the final model of clearance with the most important scaling factor; $\mathbf{T B W}$. Although the fitness of this final model was quite less than that of the basic or reduced basic models ( $\mathrm{R}$ squared = $28.07 \%$ ), it provided simple, direct and applicable equation for estimation of lithium clearance using the most significant covariate affecting clearance. $\boldsymbol{T B W}$ is found to be a better marker of body size and a better indicator of the patient's physiological conditions and metabolic capacities.

\section{Conclusions and Recommendations}

Dosage adjustment to achieve target's steady-state plasma concentration can be done using this proved correlation between lithium clearance and patient specific data (reduced Basic Model).

The final model that was obtained needs to be validated in a separate bipolar population and additional studies comparing it with other predictive methods will further elucidate its clinical utility.

\section{Acknowledgements}

This Study was completed, thanks to interns and nursing staff in the Institute of Psychiatry, Faculty of Medicine -Ain Shams University, Egypt. Also gratitude should be presented to teaching staff at Pharmacy Practice Department—Faculty of Pharmacy—Helwan University, Egypt.

\section{References}

[1] Fonseca, C.P., Montezinho, L.P. and Castro, M.M.C.A. (2012) Li+ in Bipolar Disorder-Possible Mechanisms of Its Pharmacological Mode of Action. In: Barnhill, J., Ed., Bipolar Disorder-A Portrait of a Complex Mood Disorder.

[2] Bezchlibnyk, Y. and Young, L.T. (2002) The Neurobiology of Bipolar Disorder: Focus on Signal Transduction Pathways and the Regulation of Gene Expression. Canadian Journal of Psychiatry, 47, 135-148. 
[3] Belmaker, R.H. (2004) Bipolar Disorder. New England Journal of Medicine, 351, 476-486. http://dx.doi.org/10.1056/NEJMra035354

[4] Martinez-Aran, A., et al. (2004) Cognitive Function across Manic or Hypomanic, Depressed, and Euthymic States in Bipolar Disorder. American Journal of Psychiatry, 161, 262-270. http://dx.doi.org/10.1176/appi.ajp.161.2.262

[5] Goldberg, J.F. and Roy, K.N. (2009) Chengappa, Identifying and Treating Cognitive Impairment in Bipolar Disorder. Bipolar Disorders, 11, 123-137. http://dx.doi.org/10.1111/j.1399-5618.2009.00716.x

[6] Melchior, M., et al. (2007) Work Stress Precipitates Depression and Anxiety in Young, Working Women and Men. Psychological Medicine, 37, 1119-1129. http://dx.doi.org/10.1017/S0033291707000414

[7] Hammen, C., Brennan, P.A. and Shih, J.H. (2004) Family Discord and Stress Predictors of Depression and Other Disorders in Adolescent Children of Depressed and Nondepressed Women. Journal of the American Academy of Child \& Adolescent Psychiatry, 43, 994-1002. http://dx.doi.org/10.1097/01.chi.0000127588.57468.f6

[8] American Psychiatric Association (2000) Mood Disorders. In: Diagnostic and Statistical Manual of Mental Disorders: 4th Edition: DSM-IV-TR ${ }^{\circledR}$, American Psychiatric Association, Arlington.

[9] Duman, R.S. (2002) Synaptic Plasticity and Mood Disorders. Molecular Psychiatry, 7, S29-S34. http://dx.doi.org/10.1038/sj.mp.4001016

[10] Cade, J.F. (2000) Lithium Salts in the Treatment of Psychotic Excitement 1949. Bulletin of the World Health Organization, 78, 518-520.

[11] Baldessarini, R.J., Tondo, L. and Hennen, J. (1999) Effects of Lithium Treatment and Its Discontinuation on Suicidal Behavior in Bipolar Manic-Depressive Disorders. The Journal of Clinical Psychiatry, 60, 77-84; discussion 111-116.

[12] Malhi, G.S., et al. (2013) Potential Mechanisms of Action of Lithium in Bipolar Disorder. CNS Drugs, 27, $135-153$. http://dx.doi.org/10.1007/s40263-013-0039-0

[13] Grandjean, E.M. and Aubry, J.-M. (2009) Lithium: Updated Human Knowledge Using an Evidence-Based Approach: Part II: Clinical Pharmacology and Therapeutic Monitoring. CNS Drugs, 23, 331-349. http://dx.doi.org/10.2165/00023210-200923040-00005

[14] Ward, M.E., Musa, M.N. and Bailey, L. (1994) Clinical Pharmacokinetics of Lithium. The Journal of Clinical Pharmacology, 34, 280-285. http://dx.doi.org/10.1002/j.1552-4604.1994.tb01994.x

[15] Bauer, L.A. (2008) Lithium. In: Applied Clinical Pharmacokinetics, The McGraw-Hill Companies, New York.

[16] Sproule, B.A., Hardy, B.G. and Shulman, K.I. (2000) Differential Pharmacokinetics of Lithium in Elderly Patients. Drugs \& Aging, 16, 165-177. http://dx.doi.org/10.2165/00002512-200016030-00002

[17] Thornhill, D.P. (1978) Pharmacokinetics of Ordinary and Sustained-Release Lithium Carbonate in Manic Patients after Acute Dosage. European Journal of Clinical Pharmacology, 14, 267-271. http://dx.doi.org/10.1007/BF00560460

[18] Gai, M.A.N., et al. (2000) Effect of Three Different Diets on the Bioavailability of a Sustained Release Lithium Carbonate Matrix Tablet. International Journal of Clinical Pharmacology and Therapeutics, 38, 320-326. http://dx.doi.org/10.5414/CPP38320

[19] Prien, R.F. and Caffey, E.M. (1976) Relationship between Dosage and Response to Lithium Prophylaxis in Recurrent Depression. The American Journal of Psychiatry, 133, 567-570.

[20] Stokes, P.E., Kocsis, J.H. and Arcuni, O.J. (1976) Relationship of Lithium Chloride Dose to Treatment Response in Acute Mania. Archives of General Psychiatry, 33, 1080. http://dx.doi.org/10.1001/archpsyc.1976.01770090070006

[21] Speirs, J. and Hirsch, S.R. (1978) Severe Lithium Toxicity with "Normal” Serum Concentrations. British Medical Journal, 1, 815. http://dx.doi.org/10.1136/bmj.1.6116.815

[22] Mitchell, P.B. (2001) Therapeutic Drug Monitoring of Psychotropic Medications. British Journal of Clinical Pharmacology, 52, 45-54. http://dx.doi.org/10.1111/j.1365-2125.2001.00174.x

[23] Gadallah, M.F., Feinstein, E.I. and Massry, S.G. (1988) Lithium Intoxication: Clinical Course and Therapeutic Considerations. Mineral and Electrolyte Metabolism, 14, 146-149.

[24] Mosteller, R.D. (1987) Simplified Calculation of Body-Surface Area. The New England Journal of Medicine, 317, 1098. http://dx.doi.org/10.1056/NEJM198710223171717

[25] Tietz, N.W. (1995) Clinical Guide to Laboratory Tests. WB Saunders Co., Philadelphia.

[26] Amdisen, A. (1975) Monitoring of Lithium Treatment through Determination of Lithium Concentration. Danish Medical Bulletin, 22, 277-291.

[27] Lehmann, K. and Merten, K. (1974) Die Elimination von Lithiumin Abhangigkeit vom Lebensalter bei Gesunden und Niereninsuffizienten. International Journal of Clinical Pharmacy, 10, 292-298.

[28] Reiss, R.A., et al. (1994) Lithium Pharmacokinetics in the Obese. Clinical Pharmacology \& Therapeutics, 55, $392-398$. http://dx.doi.org/10.1038/clpt.1994.47 
[29] Amdisen, A. (1977) Serum Level Monitoring and Clinical Pharmacokinetics of Lithium. Clinical Pharmacokinetics, 2, 73-92. http://dx.doi.org/10.2165/00003088-197702020-00001

[30] Platman, S.R. and Fieve, R.R. (1968) Biochemical Aspects of Lithium in Affective Disorders. Archives of General Psychiatry, 19, 659-663. http://dx.doi.org/10.1001/archpsyc.1968.01740120019003 